Original article

\title{
The uremic toxin methylguanidine increases the oxidative metabolism and accelerates the apoptosis of canine neutrophils
}

\author{
A.M. Bosco $^{a}{ }^{, *}$, B.F.M. Almeida ${ }^{\text {a }}$, P.P. Pereira ${ }^{a}$, D.B. dos Santos ${ }^{b}$, Á.J.S. Neto $^{b}$, W.L. Ferreira $^{a}$, \\ P.C. Ciarlini ${ }^{\text {a }}$ \\ a Department of Clinical Practice, Surgery and Animal Reproduction, Araçatuba College of Veterinary Medicine, São Paulo State University (UNESP), \\ Araçatuba, SP, Brazil \\ ${ }^{\mathrm{b}}$ Department of Molecular and Physical Chemistry, University of São Paulo (USP), São Carlos, SP, Brazil
}

\section{A R T I C L E I N F O}

\section{Article history:}

Received 8 September 2016

Received in revised form

24 November 2016

Accepted 26 January 2017

\section{Keywords:}

Neutrophils

Apoptosis

Oxidative metabolism

Uremic toxin

Uremia

\begin{abstract}
A B S T R A C T
We investigated the hypothesis that the increased concentration of plasma methylguanidine (MG) increases oxidative metabolism and accelerates apoptosis of neutrophils from dogs with chronic kidney disease (CKD). To achieve this, the levels of MG were quantified in healthy $(\mathrm{n}=16)$ and uremic dogs with CKD stage 4 of according to the guidelines of the International Renal Interest Society (IRIS, 2015) $(n=16)$ using high performance liquid chromatography (HPLC). To evaluate the isolated effect of MG on neutrophil oxidative metabolism and apoptosis, neutrophils isolated from 12 healthy dogs were incubated with the highest concentration of plasma MG $(0.005 \mathrm{~g} / \mathrm{L})$ observed in dogs with CKD. Neutrophil oxidative metabolism was assessed by flow cytometry, using the probes hydroethidine for superoxide production and 2',7'-dichlorofluorescein diacetate for hydrogen peroxide production, with or without phorbol myristate acetate (PMA) stimulus. Neutrophil apoptosis and viability were also evaluated in flow cytometer using the Annexin V-PE system, with or without the apoptosis-inducing effect of camptothecin. Uremic dogs presented higher concentrations of MG ( $p<0.0001)$, increased oxidative stress and primed neutrophils with higher apoptosis rate. The neutrophil abnormalities observed in vivo were also reproduced in vitro, using cells isolated from healthy dogs and incubated with MG. We obtained strong evidence that in dogs with CKD, increased MG levels contributed to oxidative stress and potentially compromised the non-specific immune response by altering the oxidative metabolism and viability of canine neutrophils.
\end{abstract}

(c) 2017 Elsevier B.V. All rights reserved.

\section{Introduction}

Neutrophils are the host's first line of cellular defense, when their oxidative metabolism is activated causing a 'respiratory burst' that generates large amounts of superoxide and derivatives, such as hydrogen peroxide, which have an important microbicidal function (Laskay et al., 2003). However, excessive production of reactive oxygen species (ROS) can accelerate cell apoptosis (Cendoroglo et al., 1999) and contribute to systemic oxidative stress (Chatterjee, 2007). This oxidative stress can compromise cell function and plays an important role in the pathogenesis of various biological processes (Reuter et al., 2010).

It is generally accepted that in human chronic kidney disease (CKD), neutrophil function is affected, contributing to increased

\footnotetext{
* Corresponding author.

E-mail address: anebosco@hotmail.com (A.M. Bosco).
}

mortality due to bacterial infections (Cohen and Hörl, 2012). Similarly to that reported in humans, recent studies have shown alterations in neutrophil oxidative metabolism and oxidative stress in dogs with CKD (Almeida et al., 2013a,b; Bosco et al., 2016; Silva et al., 2013). However, the mechanisms and relationships between neutrophil dysfunction and oxidative stress in dogs with CKD have not been fully elucidated. It has been suggested that certain uremic toxins initially activate neutrophil oxidative metabolism and the excess ROS generated induce cell apoptosis, resulting in the impairment of superoxide production and increasing the susceptibility to infections (Almeida et al., 2013b; Cendoroglo et al., 1999).

There are over 100 uremic toxins, making different contributions to neutrophil oxidative metabolism and apoptosis (Sardenberg et al., 2006). Various guanidino compounds are known to be toxic, such as methylguanidine (MG), which is not exclusively nephrotoxic, but acts as a neurotoxin (Noda and Mankura, 2009). In uremic dogs, increased levels of MG could be related to polyneuropathy (Giovannetti et al., 1969). Moreover, a positive 
correlation has been observed between MG and creatinine during uremia, which could be related to hypertension and seizures in humans (Aoyagi, 2001).

Conflicting findings have been published on the effect of MG on human neutrophil oxidative metabolism. Hirayama et al. (2000) reported no effect on neutrophil ROS production, while others authors observed inhibition of ROS production in the presence of stimulus with E. coli (Glorieux et al., 2004; Schepers et al., 2010). MG presented no effect when neutrophil oxidative metabolism was activated using phorbol myristate acetate (PMA) and $n$-formylmethionyl-leucyl-phenylalanine (fMLP) (Schepers et al., 2010).

Although the CKD is common in dogs, few studies demonstrating the effect of uremic toxins on neutrophil function are available and to date we have found no studies on MG in this species. Recently, our research group demonstrated increased plasma concentration of $p$-cresol in uremic dogs and the involvement of this toxin in neutrophil oxidative metabolism inhibition and increased apoptosis (Bosco et al., 2016). Similar studies in humans generated new important contributions to guide therapies and the development of more efficient hemodialysis membranes (Hedayat et al., 2013; Nakabayashi et al., 2011).

To expand current knowledge concerning the mechanisms involved in neutrophil dysfunction described in canine CKD, we investigated the hypothesis of associations among an increase in the concentration of plasma MG, oxidative stress, the activation of neutrophil oxidative metabolism and apoptosis in dogs.

\section{Materials and methods}

\subsection{Animal selection}

The experiment was conducted in accordance with the ethical principles of the use of animals, following approval by the Ethics Committee for Animal Experimentation of São Paulo State University (UNESP), under protocol no. FOA-00762/2012. The participation of dogs was authorized by their owners, who provided free and informed consent.

The experimental group comprised 32 adult dogs of different breeds and sexes, 16 healthy and 16 uremic dogs, all from Araçatuba City. The owners of healthy dogs from control group authorized its participation in the experiment and uremic dogs were selected at the moment of diagnosis at São Paulo State Veterinary Hospital after the tutor's consent, no treatment was performed at the time of sampling. All the dogs were submitted to the following clinical and laboratory tests: complete blood count $(\mathrm{CBC})$, reticulocytes count, urinalysis and plasma determination of total protein, albumin, urea, creatinine, total bilirubin, uric acid, cholesterol, glucose, calcium, phosphorus, urine protein:creatinine ratio (UPC).

Dogs included in the uremic group presented CKD stage 4 according to the staging system proposed by the International Renal Interest Society (IRIS, 2015), with creatinine $\geq 440 \mathrm{mmol} / \mathrm{L}$ and systemic clinical signs compatible with uremic syndrome including gastritis, mouth ulcers and uremic halitosis. Laboratory alterations included arregenerative normochromic normocytic anemia, renal azotemia, isostenuric or hyposostenuric urinary density, intense proteinuria with UPC $>1.0$ and urinary sediment characterized by the presence of hyaline casts.

Dogs vaccinated or treated in the last 30 days with drugs known to affect leukocyte function and antioxidant status were not included in the study. Considering that Araçatuba City is an endemic area for canine leishmaniasis and that this disease alters neutrophil oxidative metabolism and oxidative stress parameters (Almeida et al., 2013a,b), reagent dogs for visceral leishmaniasis by indirect ELISA method with total crude antigen of Leishmania chagasi (Lima et al., 2003) were also not included in the study.

\subsection{Study outline}

In the in vivo assays, we evaluated the effect of uremia on oxidative stress and neutrophil superoxide production and apoptosis. Plasma oxidative stress markers (total antioxidant status-TAS, lipid peroxidation measured by thiobarbituric acid reactive substancesTBARS, uric acid and total bilirubin), superoxide production, viability and apoptosis of neutrophils in healthy and uremic dogs were also correlated with the levels of MG.

To assess the isolated effects of MG on neutrophil oxidative metabolism, viability and apoptosis, we performed the in vitro assays, incubating neutrophils $\left(10^{6} / \mathrm{mL}\right)$ isolated from healthy dogs $(n=12)$ under three different treatments: neutrophils were incubated in RPMI 1640 medium alone (Control); in RPMI 1640 supplemented with $50 \%$ of uremic plasma pooled from the 16 uremic dogs; and in RPMI 1640 supplemented with $0.005 \mathrm{~g} / \mathrm{L}$ of MG (Sigma-Aldrich Co., St. Louis, USA). All the assays were conducted paired with neutrophils incubated for $3 \mathrm{~h}$ at $37^{\circ} \mathrm{C}$ in a thermocycler.

As recommended by the European Group of uremic toxins (Cohen et al., 2007), the in vitro assays were performed using the highest concentration of plasma MG observed in the uremic dogs $(0.005 \mathrm{~g} / \mathrm{L})$.

\subsection{Sample collection and laboratory analysis}

The dogs were fasted for 8-12 h prior blood collection, then $10 \mathrm{~mL}$ of blood were collected by jugular puncture, $1 \mathrm{~mL}$ was placed in $\mathrm{K}_{2}$ EDTA tube for $\mathrm{CBC}$ and $9 \mathrm{~mL}$ was collected in heparinized tubes for neutrophil isolation and to obtain plasma for biochemical analyses, measurement of oxidative stress markers and quantification of the concentrations of the uremic toxin methylguanidine.

Plasma biochemical analyzes were performed in an automated spectrophotometer (BS 200, Shenzhen Mindray Bio-Medical Electronics Co., Nanshan, China) previously calibrated with a calibrator and control serum levels I and II and using commercial reagents (Biosystems, Barcelona, Spain). The levels of the following analytes were determined: urea (UV urease/glutamate dehydrogenase enzymatic method); creatinine (alkaline picrate kinetic method); albumin (bromocresol green method); cholesterol (oxidase/peroxidase enzymatic method); total plasma protein (biuret method); bilirubin (diazotized sulfanilic method); uric acid (uricase/peroxidase enzymatic method); total calcium (cresolphthalein complexone colorimetry), inorganic phosphorus (molybdate colorimetric method); AST and ALT (IFCC kinetic method) and urinary protein (pyrogallol red method). All the biochemical reactions were performed at $37^{\circ} \mathrm{C}$, following the manufacturer's protocol.

$\mathrm{CBC}$ was performed using a veterinary automated cell counter (BC-2800 Vet, Shenzhen Mindray Bio-Medical Electronics) and reticulocyte count was performed as described previously (Thrall et al., 2004). Urine was collected by cystocentesis for urine examination. Urinary density was determined by refractometry and chemical examination was performed using commercial reagent strips (Combur test, Roche, Germany). Microscopic evaluation of urine sediment was performed following previous methodology (Fettman and Rebar, 2004) to exclude causes of proteinuria other than that of renal origin.

\subsection{Measurement of oxidative stress}

Plasma TAS was determined by colorimetry of the ABTS $\left(2,2^{\prime}-\right.$ azino-bis-3-ethylbenzthiazoline-6-sulfonic acid) cation, in which antioxidants present in the plasma inhibit the oxidation of substrates present in the reagents, preventing a change in the color of the medium and, thereby, determining the plasma total antioxidant capacity. The results were expressed as mmol Trolox/L equivalents after comparing the samples with a standard curve with several 
Trolox (Sigma-Aldrich Co., St. Louis, USA) concentrations, following methodology previously described (Erel, 2004).

Plasma lipid peroxidation was determined by quantification of TBARS using a commercial reagent (TBARS Assay Kit, ZeptoMetrix Corporation, USA) and an automatic 96-well plate reader (Readwell Touch, Robonik PVT LTD, Thane, India) at $545 \mathrm{~nm}$, following the manufacturer's recommendations.

\subsection{Chromatographic dosage methylguanidine (MG)}

Plasma samples were centrifuged at $10,000 \mathrm{rpm}$ for $10 \mathrm{~min}$ and $250 \mu \mathrm{L}$ of the supernatant were submitted to ultrafiltration at $6000 \mathrm{rpm}$ in a Centrifree ultrafiltration device with a 30,000Da cutoff (Millipore, Billerica, MA, USA). An aliquot of $100 \mu \mathrm{L}$ of ultrafiltrated sample was transferred to a microtube with $15 \mu \mathrm{L}$ of internal standard MG solution, $5.6 \mu \mathrm{L}$ of $\mathrm{KOH} 6.5 \mathrm{~mol} / \mathrm{L}$ and $9.1 \mu \mathrm{L}$ of $1.8 \mathrm{~mol} / \mathrm{L}$ ninhydrin solution. After $15 \mathrm{~min}$ of reaction, $49.9 \mu \mathrm{L}$ of ascorbic acid solution $10 \%\left(\mathrm{w} / \mathrm{v}\right.$ ) and $10 \mu \mathrm{L}$ of $\mathrm{H}_{3} \mathrm{PO}_{4} 6.45 \mathrm{~mol} / \mathrm{L}$ were added. Samples were heated at $90^{\circ} \mathrm{C}$ for $30 \mathrm{~min}$, cooled to room temperature and $10 \mu \mathrm{L}$ were injected into the Shimadzu Prominence HPLC System (20A Fluorescence detector Shimadzu RF-10AXL) with an excitation wavelength of $390 \mathrm{~nm}$ and emission wavelength of $470 \mathrm{~nm}$.

The method used was a gradient elution of $0.1 \%(\mathrm{v} / \mathrm{v})$ formic acid in water and acetonitrile, as follows (\% acetonitrile/minute): $5 \% / 0$, $23 \% / 9,50 \% / 12,5 \% / 13,5 \% / 17$ at a flow rate of $380 \mathrm{~mL} / \mathrm{min}$ and column temperature of $45^{\circ} \mathrm{C}$. The method was validated according to the orientation of the Food and Drug Administration for bioanalytical methods (FDA, 2001).

\subsection{Evaluation of neutrophil oxidative metabolism and apoptosis}

Neutrophil oxidative metabolism and apoptosis were evaluated according to previously described methodology (Bosco et al., 2016). Briefly, neutrophils isolated in double gradient were diluted $\left(10^{6} / \mathrm{mL}\right)$ in RPMI 1640 medium (Sigma-Aldrich Co., St. Louis, USA). Oxidative metabolism was evaluated by determining superoxide production with a $10 \mu \mathrm{M}$ hydroethidine probe (HE) (Invitrogen, Eugene, OR, USA) and hydrogen peroxide production with a $5 \mu \mathrm{M}$ $2^{\prime}, 7^{\prime}$-dichlorofluorescein diacetate probe (DCFH) (Sigma-Aldrich Co., St. Louis, USA), both with and without stimulus of $0.55 \mu \mathrm{M}$ PMA (Sigma-Aldrich Co., St. Louis, USA) for $10 \mathrm{~min}$ at $37^{\circ} \mathrm{C}$. The mean red (superoxide) or green (hydrogen peroxide) fluorescences were quantified by flow cytometry (Guava EasyCyte Mini ${ }^{\circledR}$, Guava Technologies, Industrial Boulevard Hayward, USA) with the acquisition of 10.000 events within the population of cells presenting neutrophil characteristics. Viability and total apoptosis (early + late apoptosis) were measured using the Annexin V-PE system (Guava nexin Kit, Guava Technologies, USA) in flow cytometry with the acquisition of 10.000 events and following the manufacturer's recommendations.

\subsection{Statistical analysis}

In the in vivo assays, the variables were tested for normality (Shapiro-Wilk test) and homoscedasticity (Bartlett's test) and the differences between groups were evaluated by the Mann-Whitney or unpaired $t$-tests. Correlation tests were performed by the Spearman test. In the in vitro assays, the variables were tested for normality (Shapiro-Wilk test) and the difference between treatments were determined by paired $t$ or Wilcoxon tests. Differences between groups were considered significant when $p<0.05$. All statistical analyses were performed using GraphPad Prism software (GraphPad Prism v.6.00 for Windows, GraphPad Software, La Jolla, CA, USA, www.graphpad.com).
Table 1

Viability and neutrophil apoptosis (mean and standard deviation) non-stimulated (NS) and in the presence of camptothecin induction (CAM) measured by the Annexin V-PE system in flow cytometry in healthy $(n=16)$ and uremic dogs $(n=16)$.

\begin{tabular}{llll}
\hline & Healthy & Uremic & p-value \\
\hline Viability NS (\%) & $98.76 \pm 0.21$ & $98.17 \pm 0.82^{\mathrm{a}}$ & 0.0087 \\
Viability CAM (\%) & $85.15 \pm 3.30$ & $77.88 \pm 4.12^{\mathrm{a}}$ & $<0.0001$ \\
Total apoptosis NS (\%) & $0.30 \pm 0.12$ & $0.48 \pm 0.27^{\mathrm{a}}$ & 0.0288 \\
Total apoptosis CAM (\%) & $7.91 \pm 2.48$ & $15.73 \pm 4.02^{\mathrm{a}}$ & $<0.0001$ \\
\hline
\end{tabular}

a Statistically significant difference by the unpaired $t$-test.

\section{Results}

\subsection{Increased concentration of plasma $M G$ and oxidative stress in uremic dogs}

The concentration of plasma MG in $87.5 \%$ of dogs from healthy group was below the detection limit $(<0.0001 \mathrm{~g} / \mathrm{L})$, so the minimum detectable value of MG was used to perform the statistical analysis. The levels of MG ranged from 0.0002 to $0.005 \mathrm{~g} / \mathrm{L}$ in uremic dogs, with mean value of $0.0013 \mathrm{~g} / \mathrm{L}$. Statistically significant increasing in MG levels was observed in uremic dogs when compared to control $(0.0001 \pm 0.0001$ vs. $0.0013 \pm 0.0015 \mathrm{~g} / \mathrm{L}, \mathrm{p}<0.0001)$ (Fig. 1$)$.

Uremic dogs also presented increased plasma lipid peroxidation $(20.12 \pm 12.20$ vs. $29.04 \pm 11.70, \mathrm{p}=0.0255)$, decreased TAS $(0.91 \pm 0.07$ vs. $0.76 \pm 0.26, p=0.040)$ and uric acid $(1.94 \pm 0.13$ vs. $0.55 \pm 0.18, \mathrm{p}<0.0001$ ) levels (Fig. 1 ). No significant correlation was observed between MG and oxidative stress markers in uremic dogs.

\subsection{Neutrophil oxidative metabolism and apoptosis in uremic} dogs and its correlation with the concentration of plasma MG

A slight, but non-significant $(56.27 \pm 18.06$ vs. $75.23 \pm 42.05$, $\mathrm{p}=0.2067$ ), increase in baseline superoxide production was observed in uremic dogs (Fig. 2A), this increase was more evident and significant $(148.3 \pm 80.40$ vs. $398.7 \pm 123.2, \mathrm{p}<0.0001)$ after neutrophil stimulation with PMA (Fig. 2B).

Simultaneously with the increase in superoxide production, decreased viability and increased apoptosis were observed in neutrophils of uremic dogs, both in the presence and absence of CAM induction (Table 1).

\subsection{In vitro effect of $M G$ and uremic plasma on oxidative metabolism and apoptosis of neutrophils from healthy dogs}

The spontaneous superoxide production of neutrophils from healthy dogs was not affected by the uremic plasma pool and the highest level of MG observed in uremic dogs ( $0.005 \mathrm{~g} / \mathrm{L}$ ) (Fig. 3A). With PMA stimulation, the uremic plasma pool decreased superoxide production $(13.10 \pm 3.96$ vs. $4.23 \pm 1.12, \mathrm{p}<0.0001)$, while MG increased neutrophil superoxide production (13.10 \pm 396 vs. $19.07 \pm 6.64, \mathrm{p}=0.001$ ) (Fig. 3A).

MG increased both spontaneous $(5.21 \pm 2.7$ vs. $7.08 \pm 2.97$, $\mathrm{p}=0.001)$ and PMA-stimulated $(10.95 \pm 7.41$ vs. $15.74 \pm 8.21$, $\mathrm{p}<0.0001$ ) neutrophil hydrogen peroxide production (Fig. 3B). In contrast, uremic plasma pool inhibited the spontaneous $(5.21 \pm 2.70$ vs. $1.13 \pm 0.76, \quad \mathrm{p}<0.001)$ and PMA-stimulated $(10.95 \pm 7.41$ vs. $1.03 \pm 0.48, \quad \mathrm{p}<0.001)$ hydrogen peroxide production (Fig. 3B).

MG and uremic plasma pool decreased viability and increased apoptosis of neutrophils from healthy dogs in vitro in nonstimulated and CAM-induced assays (Table 2). 

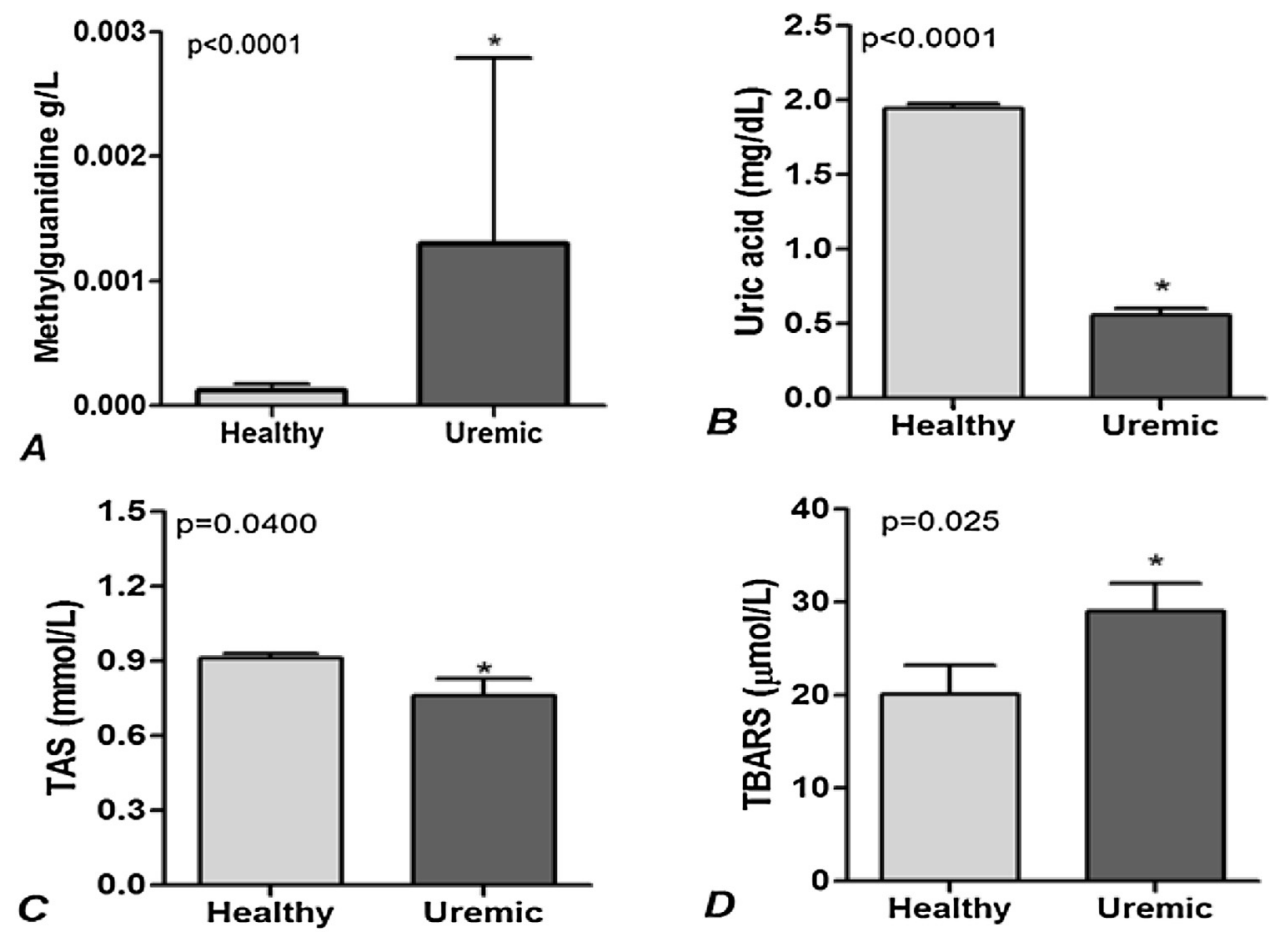

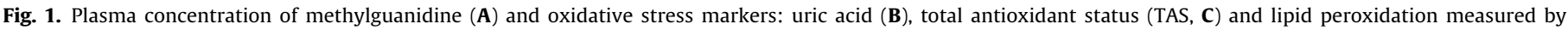
thiobarbituric acid-reactive substances (TBARS, $\mathbf{D})$ in healthy $(\mathrm{n}=16)$ and uremic $(\mathrm{n}=16)$ dogs. $\left(^{*}\right)$ Statistically significant difference by the unpaired $t$-test.

Spontaneous

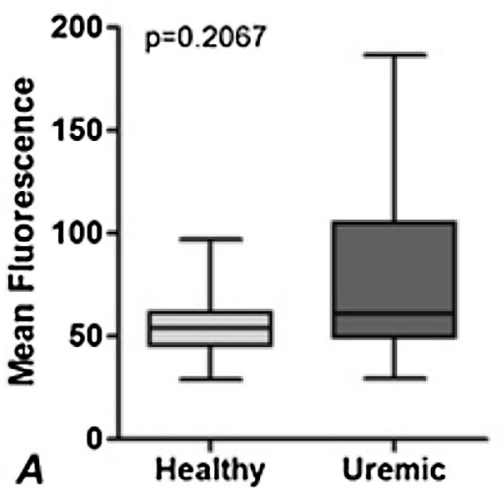

PMA stimulation

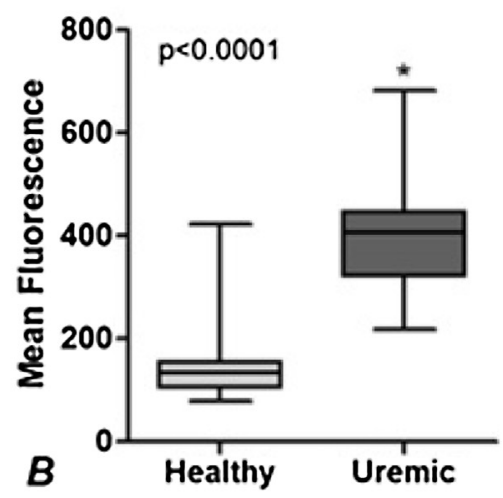

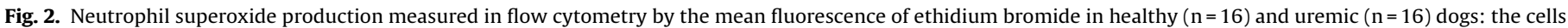

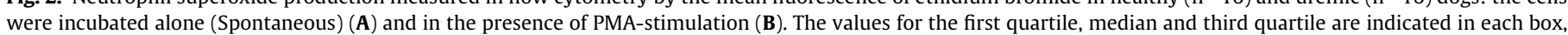
and the bars outside the box indicate minimum and maximum values. $\left(^{*}\right)$ Statistically significant difference by the Mann-Whitney test.
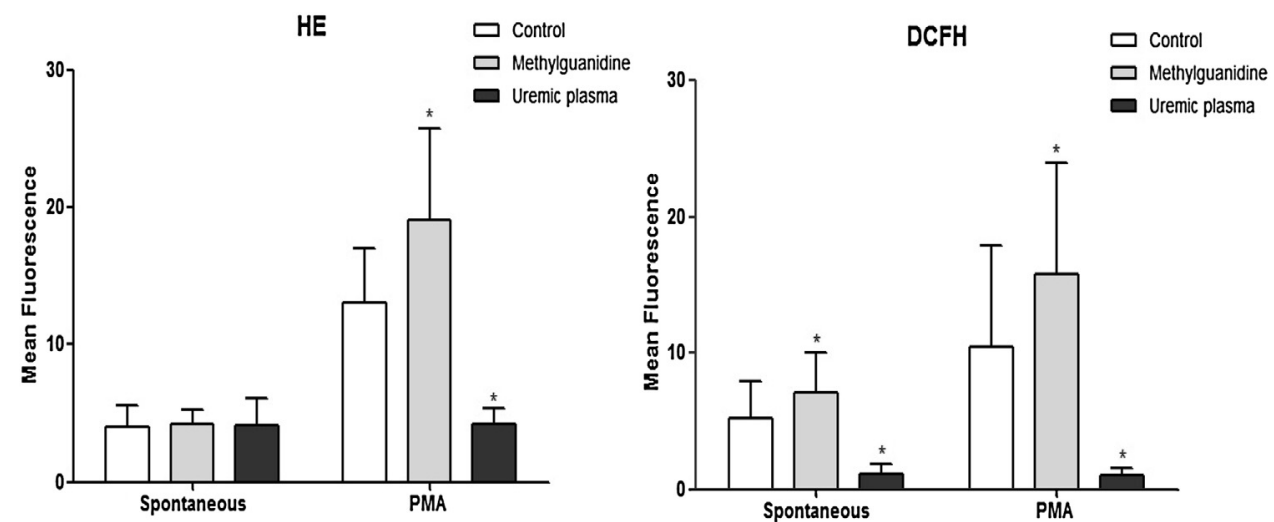

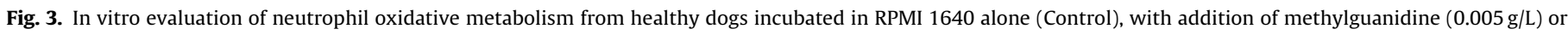

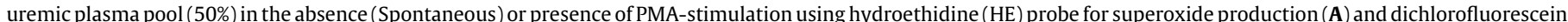
diacetate probe for hydrogen peroxide production (DCFH) $(\mathbf{B})$ in flow cytometry $(\mathrm{n}=12)$. $\left.{ }^{*}\right)$ Statistically significant difference by the unpaired $t$-test. 
Table 2

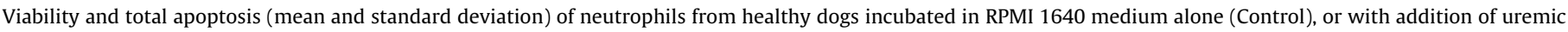
plasma pool $(50 \%)$ or methylguanidine $(0.005 \mathrm{~g} / \mathrm{L})$ in the absence (non-stimulated, NS) or presence of camptothecin induction $(\mathrm{CAM})(\mathrm{n}=12)$.

\begin{tabular}{|c|c|c|c|c|c|}
\hline Parameter & Control & Uremic plasma & p-value & Methylguanidine & p-value \\
\hline Viability NS (\%) & $99.18 \pm 0.27$ & $98.42 \pm 0.86^{\mathrm{a}}$ & 0.0164 & $98.33 \pm 1,26^{a}$ & 0.0277 \\
\hline Viability CAM (\%) & $79.91 \pm 5.18$ & $64.18 \pm 10.43^{a}$ & 0.0011 & $42.65 \pm 5,22^{a}$ & 0.0107 \\
\hline Total apoptosis NS (\%) & $0.38 \pm 0.20$ & $1.36 \pm 0.79^{\mathrm{a}}$ & 0.0020 & $0.68 \pm 0,47^{\mathrm{a}}$ & 0.0464 \\
\hline Total apoptosis CAM (\%) & $19.38 \pm 4.49$ & $34.53 \pm 10.80^{\mathrm{a}}$ & 0.0018 & $24.73 \pm 4,97^{a}$ & 0.0093 \\
\hline
\end{tabular}

a Statistically significant difference by the unpaired $t$-test.

\section{Discussion}

The hematological and biochemical profiles of healthy dogs were in accordance with the normal values of the species (Thrall et al., 2004), certifying the good health of these dogs. Laboratory and clinical changes observed in uremic dogs were similar to those recently described (Almeida et al., 2013b; Bosco et al., 2016) and were equivalent to stage 4 , according to IRIS staging (IRIS, 2015).

The hydroxyl radical produced from superoxide by neutrophil oxidative metabolism appears to play an important role in MG biosynthesis, such that it has been proposed that the increase in MG levels may reflect the increased ROS production in humans (Huang et al., 2003). Studies evaluating the increase of MG in dog CKD are rare, dogs submitted to ureteral ligation presented the clinical signs of CKD and high blood concentration of MG, with an average of $0.0021 \mathrm{~g} / \mathrm{L}$ (Giovannetti et al., 1973). A single clinical study reports the increase in plasma MG in dogs with CKD $(0.0001 \mathrm{~g} / \mathrm{L})$ compared with healthy dogs $(0.000015 \mathrm{~g} / \mathrm{L})$ (Brooks et al., 1989).

In this study, the oxidative stress of uremic dogs was characterized by increased plasma lipid peroxidation, decreased TAS and uric acid, corroborating that reported by recent studies (Almeida et al., 2013b; Bosco et al., 2016; Silva et al., 2013). Increased MG and decreased TAS have also been reported in human patients; after hemodialysis, decreased MG and increased TAS was observed, suggesting a possible action of this toxin on oxidative stress (Huang et al., 2003). However, we were unable to establish a direct correlation between oxidative stress markers and MG in uremic dogs.

The slight higher spontaneous superoxide production and the large increase after activation with PMA indicates the primed state of neutrophils from uremic dogs, as previously described (Almeida et al., 2013b). It has been proposed that the primed state of human neutrophils can be caused by the presence of uremic toxins (McLeish et al., 1996). The persistence of the primed state of neutrophils favors oxidative stress and accelerates neutrophil apoptosis (Mazor et al., 2008), which could explain the higher rate of neutrophil apoptosis observed in the uremic group, corroborating reports from other studies (Almeida et al., 2013b; Bosco et al., 2016).

It is believed that in the initial stage of CKD, activation of neutrophil oxidative metabolism occurs, generating a large amount of ROS that exceeds the cellular antioxidant capacity, causing injury to vital cellular structures and accelerating the process of apoptosis (Cendoroglo et al., 1999). It has also been proposed that primed neutrophils from dogs with CKD can contribute to oxidative stress, and consequently affect the function and viability of these cells (Almeida et al., 2013b).

Several uremic toxins affect neutrophil function and guanidino compounds have received special attention. Reports on the effects of MG on neutrophil function are scarce and contradictory (Glorieux et al., 2004). It have been reported that MG inhibits neutrophil oxidative metabolism when activated by $E$. coli in humans, a fact not observed when the activation was performed with PMA or fMLP (Hirayama et al., 2000; Schepers et al., 2010). Guanidino compounds, such as guanidine-acetic acid, inhibit in vitro neutrophil oxidative metabolism from dogs (Pereira et al., 2015). Thus, we compared the in vitro effect of MG and uremic plasma, and showed that this toxin alone increased ROS production (superoxide and hydrogen peroxide) and accelerated the apoptosis of neutrophils from healthy dogs.

The primed state and higher apoptosis rate observed in neutrophils from uremic dogs were reproduced in vitro using neutrophils isolated from healthy dogs incubated with MG, but not with uremic plasma. The production of superoxide and hydrogen peroxide decreased in neutrophils of healthy dogs incubated with uremic plasma, a finding also been reported in other studies (Bosco et al., 2016).

In vitro studies with mice have shown that MG itself generates the hydroxyl radical, a derivative of hydrogen peroxide (Noda and Mankura, 2009). MG also enhances the pro-apoptotic effect of hydrogen peroxide on human glial cells in vitro (Marzocco et al., 2010). To our knowledge, this is the first evidence that the concentration of MG in dogs can promote the activation of neutrophil oxidative metabolism, increasing the production of superoxide and hydrogen peroxide, thereby contributing to increased oxidative stress and apoptosis in neutrophils. These results support the hypothesis that MG contributes to oxidative stress and may compromise the non-specific immune response of dogs with CKD.

This study should be considered a starting point for further investigations concerning the mechanisms involved in oxidative stress and immune suppression in dogs with CKD. Although the changes observed in dogs with CKD have been partially reproduced in vitro, the isolated effect of MG needs to be better elucidated in order to guide future therapies that minimize the undesirable effects of this toxin.

\section{Conclusions}

The accumulation of methylguanidine in dogs with CKD favors oxidative stress and may impair non-specific immunity by altering neutrophil oxidative metabolism and apoptosis.

\section{Conflict of interest statement}

None of the authors of this paper has a financial or personal relationship with other people or organizations that could inappropriately influence or bias the content of the paper.

\section{Acknowledgement}

The authors are grateful to the São Paulo Research Foundation (FAPESP) for the Masters grant (Proc. 2011/16018-0) and for funding the project (Proc. 2011/18746-3).

\section{References}

Almeida, B.F., Narciso, L.G., Bosco, A.M., Pereira, P.P., Braga, E.T., Avanço, S.V., Marcondes, M., Ciarlini, P.C., 2013a. Neutrophil dysfunction varies with the stage of canine visceral leishmaniosis. Vet. Parasitol. 196, 6-12.

Almeida, B.F., Narciso, L.G., Melo, L.M., Preve, P.P., Bosco, A.M., Lima, V.M., Ciarlini, P.C., 2013b. Leishmaniasis causes oxidative stress and alteration of oxidative metabolism and viability of neutrophils in dogs. Vet. J. 198, 599-605.

Aoyagi, K., 2001. Significance of reactive oxygen in kidney disease elucidated by uremic toxins. J. Artif. Organs 4, 3-7. 
Bosco, A.M., Pereira, P.P., Almeida, B.F., Narciso, L.G., Dos Santos, D.B., Santos-Neto, Á., Ferreira, W.L., Ciarlini, P.C., 2016. Free p-cresol alters neutrophil function in dogs. Artif. Organs 40, 480-488.

Brooks, D.P., Rhodes, G.R., Woodward, P., Boppana, V.K., Mallon, F.M., Griffin, H.E. Kinter, L.B., 1989. Production of methylguanidine in dogs with acute and chronic renal failure. Clin. Sci. (Lond.) 77, 637-641.

Cendoroglo, M., Jaber, B.L., Balakrishnan, V.S., Perianayagam, M., King, A.J., Pereira, B.J., 1999. Neutrophil apoptosis and dysfunction in uremia. J. Am. Soc. Nephrol 10, 93-100.

Chatterjee, P.K., 2007. Novel pharmacological approaches to the treatment of renal ischemia-reperfusion injury: a comprehensive review. Naunyn. Schmiedebergs Arch. Pharmacol. 376, 1-43.

Cohen, G., Hörl, W.H., 2012. Immune dysfunction in uremia-an update. Toxins (Basel) 4, 962-990

Cohen, G., Glorieux, G., Thornalley, P., Schepers, E., Meert, N., Jankowski, J., Jankowski, V., Argiles, A., Anderstam, B., Brunet, P., Cerini, C., Dou, L., Deppisch R., Marescau, B., Massy, Z., Perna, A., Raupachova, J., Rodriguez, M., Stegmayr, B., Vanholder, R., Hörl, W.H., European Uremic Toxin Work Group (EUTox), 2007. Review on uraemic toxins III: recommendations for handling uraemic retention solutes in vitro-towards a standardized approach for research on uraemia. Nephrol. Dial. Transplant. 22, 3381-3390.

Erel, O., 2004. A novel automated direct measurement method for total antioxidant capacity using a new generation, more stable ABTS radical cation. Clin. Biochem. 37, 277-285.

Fettman, M.J., Rebar, A., 2004. Laboratory evaluation of renal function. In: Thrall, M.A., Baker, D.C., Lassen, E.D., DeNicola, D., J, F.M., Lassen, E.D., Rebar, A., Weiser, G. (Eds.), Veterinary Hematology and Clinical Chemistry. Lippincott Willians \& Wilkins, Philadelphia, pp. 301-314, cap.21.

Giovannetti, S., Biagini, M., Balestri, P.L., Navalesi, R., Giagnoni, P., De Matteis, A Ferro-Milone, P., Perfetti, C., 1969. Uraemia-like syndrome in dogs chronically intoxicated with methylguanidine and creatinine. Clin. Sci. 36, 445-452.

Giovannetti, S., Balestri, P.L., Barsotti, G., 1973. Methylguanidine in uremia. Arch. Intern. Med. 131, 709-713.

Glorieux, G.L., Dhondt, A.W., Jacobs, P., Van Langeraert, J., Lameire, N.H., De Deyn, P.P., Vanholder, R.C., 2004. In vitro study of the potential role of guanidines in leukocyte functions related to atherogenesis and infection. Kidney Int. 65, 2184-2192.

Hedayat, A., Peace, R., Elmoselhi, H., Shoker, A., 2013. Study of uremic toxin fluxes across nanofabricated hemodialysis membranes using irreversible thermodynamics. Comput. Struct. Biotechnol. J. 6, e201303005.

Hirayama, A., Noronha-Dutra, A.A., Gordge, M.P., Neild, G.H., Hothersall, J.S., 2000. Inhibition of neutrophil superoxide production by uremic concentrations of guanidino compounds. J. Am. Soc. Nephrol. 11, 684-689.

Huang, K.C., Yang, C.C., Lee, K.T., Chien, C.T., 2003. Reduced hemodialysis-induced oxidative stress in end-stage renal disease patients by electrolyzed reduced water. Kidney Int. 64, 704-714.

IRIS, 2015. IRIS Staging of Chronic Renal Disease. International Renal Interest Society, http://www.iris-kidney.com/pdf/staging-of-ckd.pdf (Accessed 15 July 2016)
Laskay, T., van Zandbergen, G., Solbach, W., 2003. Neutrophil granulocytes-Trojan horses for Leishmania major and other intracellular microbes? Trends Microbiol. 11, 210-214.

Lima, V.M., Gonçalves, M.E., Ikeda, F.A., Luvizotto, M.C., Feitosa, M.M., 2003. Anti-leishmania antibodies in cerebrospinal fluid from dogs with visceral leishmaniasis. Braz. J. Med. Biol. Res. 36, 485-489.

Marzocco, S., Popolo, A., Bianco, G., Pinto, A., Autore, G., 2010. Pro-apoptotic effect of methylguanidine on hydrogen peroxide-treated rat glioma cell line. Neurochem. Int. 57, 518-524.

Mazor, R., Shurtz-Swirski, R., Farah, R., Kristal, B., Shapiro, G., Dorlechter, F. Cohen-Mazor, M., Meilin, E., Tamara, S., Sela, S., 2008. Primed polymorphonuclear leukocytes constitute a possible link between inflammation and oxidative stress in hyperlipidemic patients. Atherosclerosis 197, 937-943.

McLeish, K.R., Klein, J.B., Lederer, E.D., Head, K.Z., Ward, R.A., 1996. Azotemia, TNF alpha, and LPS prime the human neutrophil oxidative burst by distinct mechanisms. Kidney Int. 50, 407-416.

Nakabayashi, I., Nakamura, M., Kawakami, K., Ohta, T., Kato, I., Uchida, K., Yoshida, M., 2011. Effects of synbiotic treatment on serum level of p-cresol in haemodialysis patients: a preliminary study. Nephrol. Dial. Transplant. 26, 1094-1098.

Noda, Y., Mankura, M., 2009. Inhibitory effect of antioxidants on hydroxyl radical generation from methylguanidine: an ESR study. Neurochem. Res. 34, 734-738.

Pereira, P.P., Bosco, A.M., de Almeida, B.F.M., Narciso, L.G., Ciarlini, P.C., 2015. A toxina urêmica ácido guanidinicoacético inibe o metabolismo oxidativo dos neutrófilos de cães. Ciência Animal Brasileira 16, 560-566.

Reuter, S., Gupta, S.C., Chaturvedi, M.M., Aggarwal, B.B., 2010. Oxidative stress, inflammation, and cancer: how are they linked? Free Radic. Biol. Med. 49, 1603-1616.

Sardenberg, C., Suassuna, P., Andreoli, M.C., Watanabe, R., Dalboni, M.A., Manfredi, S.R., dos Santos, O.P., Kallas, E.G., Draibe, S.A., Cendoroglo, M., 2006. Effects of uraemia and dialysis modality on polymorphonuclear cell apoptosis and function. Nephrol. Dial. Transplant. 21, 160-165.

Schepers, E., Glorieux, G., Dou, L., Cerini, C., Gayrard, N., Louvet, L., Maugard, C., Preus, P., Rodriguez-Ortiz, M., Argiles, A., Brunet, P., Cohen, G., Jankowski, J., Jankowski, V., Massy, Z., Rodriguez, M., Vanholder, R., European Uremic Toxin Work Group (EUTox), 2010. Guanidino compounds as cause of cardiovascular damage in chronic kidney disease: an in vitro evaluation. Blood Purif. 30, 277-287.

Silva, A.C., de Almeida, B.F., Soeiro, C.S., Ferreira, W.L., de Lima, V.M., Ciarlini, P.C., 2013. Oxidative stress, superoxide production, and apoptosis of neutrophils in dogs with chronic kidney disease. Can. J. Vet. Res. 77, 136-141.

Thrall, M.A., Baker, D.C., Lassen, E.D., DeNicola, D., J, F.M., Lassen, E.D., Rebar, A. Weiser, G., 2004. Veterinary Hematology and Clinical Chemistry. Lippincott Williams \& Wilkins, Philadelphia.

U.S. Food and Drug Administration (FDA), 2001. Guidance for Industry, Bioanalytical Method Validation, http://www. fda. gov/cvm. (Accessed 02 July 2016). 